\title{
Tracheobronchomalacia due to amyloidosis in a patient with rheumatoid arthritis
}

\author{
Franktien Turkstra • Rico N. P. M. Rinkel • \\ Hagen Biermann • Paul van der Valk • \\ Alexandre E. Voskuyl
}

Received: 27 October 2007 /Revised: 27 December 2007 / Accepted: 1 January 2008 / Published online: 19 January 2008

(C) Clinical Rheumatology 2008

\begin{abstract}
In this case report, we describe a patient with longstanding rheumatoid arthritis who developed tracheobronchomalacia with fatal outcome. Despite negative antemortem biopsies of abdominal fat and tongue, amyloid was found postmortem in the trachea and appeared to be associated with tracheobronchomalacia.
\end{abstract}

Keywords Amyloidosis · Kidney · Rheumatoid arthritis ·

Tracheobronchomalacia

\section{Introduction}

Amyloidosis in longstanding chronically active rheumatoid arthritis (RA) is a well-known complication of this disease [1]. We present a RA patient with amyloidosis, albeit with a unique presenting symptom and unexpected organ involvement.

\section{Case report}

The patient was a 69-year-old female with seropositive erosive RA, who had not been treated with diseasemodifying antirheumatic drugs since 1984. In March 2005, she presented to another hospital because of progressive dyspnea of several hours, due to a bronchopneumonia. After 2 days, she developed a stridor. Otolaryngological examination results showed diffuse swelling of the tongue, pharynx and neck, and an extensive tracheobronchomalacia. Treatment consisted of intubation, antibiotics, diuretic therapy, and corticosteroids. She was admitted to our Intensive Care Unit because of progressive swelling and respiratory insufficiency due to tracheobronchomalacia. Physical examination results revealed extensive swelling and a remarkable protrusion of the tongue. Typical rheumatoid joint deformities and rheumatoid nodules were present but no active arthritis. Laboratory investigations revealed a normocytic anemia and a renal insufficiency. The measured creatinine clearance was $12 \mathrm{ml} / \mathrm{min}$, with a proteinuria of $0.35 \mathrm{~g} / 24 \mathrm{~h}$. Computed

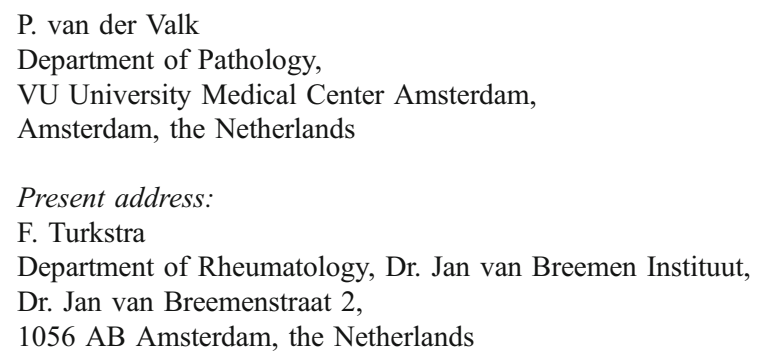

F. Turkstra $(\bowtie) \cdot$ A. E. Voskuyl

Department of Rheumatology,
Amsterdam, the Netherlands

e-mail: f.turkstra@janvanbreemen.nl

R. N. P. M. Rinkel

Department of Otolaryngology-Head and Neck Surgery,

VU University Medical Center

Amsterdam, the Netherlands

H. Biermann

Department of Intensive Care,

VU University Medical Center Amsterdam,

Amsterdam, the Netherlands 


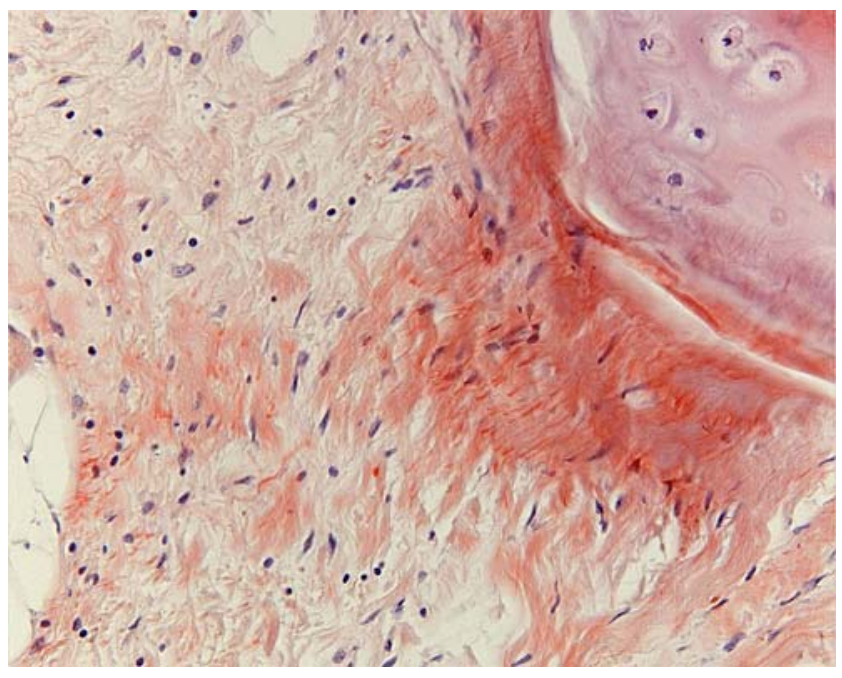

Fig. 1 Amyloidosis of the trachea in Congo red

tomography scan of thorax and neck provided no explanation for the tracheobronchomalacia. Clinical suspicion of amyloidosis was not confirmed by biopsies of abdominal fat tissue and of the tongue. Ultimately, the patient succumbed to multiple organ failure. Autopsy revealed AA amyloid in the trachea (Fig. 1), spleen, liver, and perivascularly in the kidneys.

\section{Discussion}

Amyloidosis of the trachea is a rare but known disorder, even rarer in patients with systemic diseases (such as Sjogren syndrome) but never associated with tracheobronchomalacia [2-4]. We report the first case of an RA patient with tracheobronchomalacia due to AA amyloidosis of the trachea. The diagnosis was only confirmed at postmortem, although several biopsies had been done in life to confirm the clinical suspicion. We refrained from rectal and kidney biopsies because of the relatively high risk of complications associated with these procedures; the absence of diarrhea and the low level of proteinuria suggested a relatively low pretest probability. Therefore, the finding of renal amyloid was surprising. Recently, Uda et al. [5] described a prospective cohort study of patients with RA without clinical signs of kidney disease and amyloidosis of the kidney. At follow-up, the group of patients with amyloid deposition confined to the perivascular region continued to have little proteinuria and a good renal prognosis, compared to those with amyloid deposition in the glomeruli who developed rapidly deteriorating renal function. This patient, however, does show that the disease course in RA patients with perivascular amyloid deposition in the kidney is not benign and may be associated with fatal complications elsewhere.

In conclusion, tracheobronchomalacia can be due to amyloid deposition in patients with RA. Amyloidosis should be considered in RA patients with a small amount of proteinuria, and a renal biopsy should be performed, especially as accumulating evidence shows that patients with amyloidosis have a better prognosis when the ongoing inflammation is effectively suppressed [1].

Acknowledgements The authors would like to thank John MacFarlane M.D., Ph.D. (rheumatologist) for his help with the manuscript.

Disclosures None

\section{References}

1. Lachmann HJ, Goodman HJ, Gilbertson JA et al (2007) Natural history and outcome in systemic AA amyloidosis. N Engl J Med 356:2361-2371

2. O'Regan A, Fenlon HM, Beamis JF Jr et al (2000) Tracheobronchial amyloidosis. The Boston University experience from 1984 to 1999. Medicine (Baltimore) 79:69-79

3. Xu L, Cai BQ, Zhong X et al (2005) Respiratory manifestations in amyloidosis. Chin Med J (Engl) 118:2027-2033

4. Carden KA, Boiselle PM, Waltz DA et al (2005) Tracheomalacia and tracheobronchomalacia in children and adults: an in-depth review. Chest 127:984-1005

5. Uda H, Yokota A, Kobayashi K et al (2006) Two distinct clinical courses of renal involvement in rheumatoid patients with AA amyloidosis. J Rheumatol 33:1482-1487 